Alexander Zweig, MD; Carl Tunink, MD; Laura Morris, MD, MSPH

Department of Family and Community Medicine, University of Missouri, Columbia

DEPUTY EDITOR Shailendra Prasad, MBBS, MPH

University of Minnesota North Memorial Family Medicine Residency Program, Minneapolis doi: $10.12788 /$ jfp.0320

\title{
Skip that repeat DXA scan in these postmenopausal women
}

\author{
Repeat bone density measurement offers no advantage \\ in predicting fracture risk in postmenopausal women \\ who do not have osteoporosis.
}

\section{PRACTICE CHANGER}

Do not routinely repeat bone density testing 3 years after initial screening in postmenopausal patients who do not have osteoporosis.

STRENGTH OF RECOMMENDATION

A: Based on several large, good-quality prospective cohort studies ${ }^{1}$

Crandall CJ, Larson J, Wright NC, et al. Serial bone density measurement and incident fracture risk discrimination in postmenopausal women. JAMA Intern Med. 2020;180:1232-1240. doi: 10.1001/jamainternmed.2020.2986

\section{ILLUSTRATIVE CASE}

A 70-year-old White woman with a history of type 2 diabetes and a normal body mass index (BMI) presents to your office for a preventive care exam. She is otherwise doing well, without concerns. Her first dual-energy x-ray absorptiometry (DXA) scan, completed at age 67, demonstrated normal bone density. Should you recommend a repeat DXA scan today?

A s many as 1 in 2 postmenopausal women are at risk for an osteoporosis-related fracture. ${ }^{2}$ Each year, about 2 million fragility fractures occur in the United States. ${ }^{2,3}$ The US Preventive Services Task Force (USPSTF) recommends bone mineral density (BMD) measurement in all women ages 65 years and older, as well as in younger postmenopausal women with certain clinical risk factors. ${ }^{4}$ The USPSTF does not make a recommendation regarding the interval for follow-up BMD testing.
Two prospective cohort studies determined that repeat BMD testing 4 to 8 years after baseline screening did not improve fracture risk prediction. ${ }^{5,6}$ Limitations of these studies included no analysis of high-risk subgroups, as well as failure to include many younger postmenopausal women in the studies. ${ }^{5,6}$ An additional longitudinal study that followed postmenopausal women for up to 15 years estimated that the interval for at least $10 \%$ of women to develop osteoporosis after initial screening was more than 15 years for women with normal BMD and about 5 years for those with moderate osteopenia. ${ }^{7}$

\section{STUDY SUMMARY}

\section{No added predictive benefit} found in 3-year repeat scan

The current study examined data from the Women's Health Initiative Extension 1 Study, a large prospective cohort that included a broader range of postmenopausal women $(\mathrm{N}=7419)$ than the previous studies. The purpose of this study was to determine if a second BMD measurement, about 3 years after the baseline BMD screening, would be useful in predicting risk for major osteoporotic fracture (MOF), compared with baseline BMD measurement alone. It analyzed data from prespecified subgroups defined by age, BMI, race/ethnicity, presence or absence of diabetes, and baseline BMD T score. ${ }^{1}$

Study participants averaged 66 years of age, with a mean BMI of 29 , and $23 \%$ were non-White. In addition, $97 \%$ had either nor- 
mal BMD or osteopenia ( $\mathrm{T}$ score $\geq-2.4$ ). Participants were excluded from the study if they had been treated with bone-active medications other than vitamin D and calcium, reported a history of MOF (fracture of the hip, spine, radius, ulna, wrist, upper arm, or shoulder) at baseline or between BMD tests, missed follow-up visits after the Year 3 BMD scan, or had missing covariate data. Participants self-reported fractures on annual patient questionnaires, and hip fractures were confirmed through medical records.

During the mean follow-up period of 9 years after the second BMD test, 139 women $(1.9 \%)$ had 1 or more hip fractures, and 732 women $(9.9 \%)$ had 1 or more MOFs.

Area under the receiver operating characteristic curve (AU-ROC) values for baseline BMD screening and baseline plus 3-year BMD measurement were similar in their ability to discriminate between women who had a hip fracture or MOF and women who did not. AU-ROC values communicate the usefulness of a diagnostic or screening test. An AUROC value of 1 would be considered perfect ( $100 \%$ sensitive and $100 \%$ specific), whereas an AU-ROC of 0.5 suggests a test with no ability to discriminate at all. Values between 0.7 and 0.8 would be considered acceptable, and those between 0.8 and 0.9 , excellent.

The AU-ROCs in this study were 0.71 (95\% CI, 0.67-0.75) for baseline total hip BMD, 0.61 (95\% CI, 0.56-0.65) for change in total hip BMD between baseline and 3-year BMD scan, and 0.73 (95\% CI, 0.69-0.77) for the combined baseline total hip BMD and change in total hip BMD. For femoral neck and lumbar spine BMD, AU-ROC values demonstrated comparable discrimination of hip fracture and MOF as those for total hip BMD. The AU-ROC values among age subgroups ( $<65$ years, $65-74$ years, and $\geq 75$ years) were also similar. Associations between change in bone density and fracture risk did not change when adjusted for factors such as BMI, race/ ethnicity, diabetes, or baseline BMD.

\section{WHAT"S NEW}

\section{Results can be applied} to a wider range of patients

This study found that for postmenopausal women, a repeat BMD measurement obtained 3 years after the initial assessment did not improve risk discrimination for hip fracture or MOF beyond the baseline BMD value and should not be routinely performed. Additionally, evidence from this study allows this recommendation to apply to younger postmenopausal women and a variety of high-risk subgroups.

\section{CAVEATS}

\section{Possible bias due to self-reporting of fractures}

This study suggests that for women without a diagnosis of osteoporosis at initial screening, repeat testing is unlikely to affect future risk stratification. Repeat BMD testing should still be considered when the results are likely to influence clinical management.

However, an important consideration is that fractures were self-reported in this study, introducing a possible source of bias. Additionally, although this study supports foregoing repeat screening at a 3-year interval, there is still no agreed-upon determination of when (or if) to repeat BMD screening in women without osteoporosis.

A large subset of the study population was younger than 65 (44\%), the age when family physicians typically recommend screening for osteoporosis. However, the age-adjusted AUROC values for fracture risk prediction were the same, and this should not invalidate the conclusions for the study population at large.

\section{CHALLENGES TO IMPLEMENTATION}

\section{No challenges seen}

We see no challenges in implementing this recommendation.

\section{ACKNOWLEDGEMENT}

The PURLs Surveillance System was supported in part by Grant Number UL1RR024999 from the National Center for Research Resources, a Clinical Translational Science Award to the University of Chicago. The content is solely the responsibility of the authors and does not necessarily represent the official views of the National Center for Research Resources or the National Institutes of Health.

Copyright @ 2021 . The Family Physicians Inquiries Network. All rights reserved.

References

1. Crandall CJ, Larson J, Wright NC, et al. Serial bone density measurement and incident fracture risk discrimination in post- 
menopausal women. JAMA Intern Med. 2020;180:1232-1240. doi: 10.1001/jamainternmed.2020.2986

2. US Preventive Services Task Force. Screening for osteoporosis: US Preventive Services Task Force recommendation statement Ann Intern Med. 2011;154:356-364. doi: 10.7326/0003-4819-1545-201103010-00307

3. Burge R, Dawson-Hughes B, Solomon DH, et al. Incidence and economic burden of osteoporosis-related fractures in the United States, 2005-2025. I Bone Miner Res. 2007;22:465-475. doi: $10.1359 /$ jbmr.061113

4. US Preventive Services Task Force; Curry SJ, Krist AH, Owens DK, et al. Screening for osteoporosis to prevent fractures: US Preventive Services Task Force recommendation statement. JAMA. 2018;319:2521-2531. doi: 10.1001/jama.2018.7498

5. Hillier TA, Stone KL, Bauer DC, et al. Evaluating the value of repeat bone mineral density measurement and prediction of fractures in older women: the study of osteoporotic fractures. Arch Intern Med. 2007;167:155-160. doi: 10.1001/archinte.167.2.155

6. Berry SD, Samelson EJ, Pencina MJ, et al. Repeat bone mineral density screening and prediction of hip and major osteoporotic fracture. JAMA. 2013;310:1256-1262. doi: 10.1001/ jama.2013.277817

7. Gourlay ML, Fine JP, Preisser JS, et al; Study of Osteoporotic Fractures Research Group. Bone-density testing interval and transition to osteoporosis in older women. $N$ Engl J Med. 2012;366: 225-233. doi: 10.1056/NEJMoa1107142

\section{WE WANT TO HEAR FROM YOU!}

Have a comment on an article, editorial, or department? You can send it by:

1. E-MAIL:jfp.eic@gmail.com

2. FAX: 973-206-9251 or

3. MAIL:The Journal of Family Practice, 7 Century Drive, Suite 302, Parsippany, NJ 07054

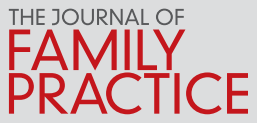

LETTERS SHOULD BE 200 WORDS OR LESS. THEY WILL BE EDITED PRIOR TO PUBLICATION.

\section{Visit us @}

(y) mdedge.com/ familymedicine

\section{1) ACIP simplifies adult} vaccinations for HepB and pneumonia

Doug Campos-Outcalt, MD, MPA

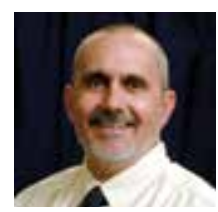

\section{ONLINE EXCLUSIVES}

- CLINICAL INQUIRIES

Does inadequate sleep increase obesity risk in children?

- PHOTO ROUNDS

White ankle scars

- RESIDENTS' RAPID REVIEW A 5-question monthly quiz to help you prepare for the family medicine (re)certification exam. This month: EKG challenge

\section{PHOTO ROUNDS FRIDAY}

Test your diagnostic skills at www.mdedge.com/familymedicine/ photo-rounds

\section{PLUS}

Today's headlines in family medicine

GET UPDATES FROM US ON
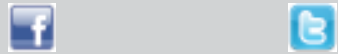

www.facebook.com/JFamPract http://twitter.com/JFamPract

www.mdedge.com/

familymedicine 